\title{
Probing extended modes on disordered plasmonic networks by wavefront shaping
}

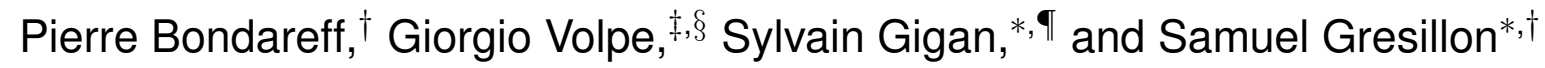 \\ Institut Langevin, University Pierre et Marie Curie, ESPCI ParisTech, CNRS UMR7587, \\ 1 rue Jussieu, 75238 Paris Cedex 05, France, Department of Chemistry, University College \\ London, 20 Gordon Street, London WC1H OAJ, UK, and Laboratoire Kastler Brossel, \\ UMR8552 of CNRS and University Pierre et Marie Curie, Ecole Normale Supérieure and \\ College de France, 24 rue Lhomond, 75005 Paris, France
}

\begin{abstract}
We experimentally study the optical field distribution on disordered plasmonic networks by far-field wavefront shaping. We observe non-local fluctuations of the field intensity mediated by plasmonic modes up to a distance of $10 \mu \mathrm{m}$ from the excitation area. In particular we quantify the spatial extent of these fluctuations as a function of the metal filling fraction in the plasmonic network and we identify a clear increase around percolation due to the existence of extended plasmonic modes. This paves the way towards far-field coherent control of plasmonic modes on similar disordered plasmonic networks. We expect these results to be relevant for quantum networks, coherent control and light matter interactions in such disordered films where long range interactions are critical.
\end{abstract}

\footnotetext{
${ }^{*}$ To whom correspondence should be addressed

${ }^{\dagger}$ Institut Langevin

${ }^{\ddagger}$ Current affiliation: University College London

"Laboratoire Kastler Brossel

${ }^{\S}$ Laboratoire Kastler Brossel, UMR8552 of CNRS and University Pierre et Marie Curie, Ecole Normale Supérieure and College de France, 24 rue Lhomond, 75005 Paris, France
} 


\section{Introduction}

The field of plasmonics offers a unique route for bridging the gap between photonics and the nanometer scale with typical subdiffraction features. ${ }^{1,2}$ In fact, plasmonic structures can efficiently couple propagating light and concentrate it into nanometric volumes, and viceversa. ${ }^{1,2}$ While the location of these hot spots is mostly determined by the geometry of the plasmonic system, their dynamical reconfiguration has potential for applications in sensing, ${ }^{3}$ photovoltaics, ${ }^{4}$ quantum networks,${ }^{5}$ nanocircuitry, ${ }^{6}$ metamaterials,${ }^{7}$ and superresolution microscopy. ${ }^{8}$ Several strategies borrowed by the field of coherent control have been suggested to reach this goal. ${ }^{9-15}$ But selectivity is limited to a few modes predefined by the sample structure and typically requires advanced fabrication.

Disordered plasmonics networks, such as dielectrics and metal-dielectric composites, provide a new class of photonic materials to control light propagation and enhance the interaction of light with matter down to the single molecule level. ${ }^{16-18}$ Disordered semicontinuous metal films, in particular, exhibit intrinsic optical properties that strongly differ from those of bulk metals or ensembles of single nanoparticles, such as broadband absorption ${ }^{19-21}$ and giant field fluctuations. ${ }^{22-24}$ Previous experimental work has tried to quantify the spatial extent of these modes in the near-field, ${ }^{25-31}$ where non radiative components (dark modes) dominate over radiative ones (bright modes). ${ }^{29,32,33}$ It is not yet clear, therefore, to which extent the plasmonic modes of these systems can be actively controlled from the far-field by means of coherent control techniques, thus allowing for the dynamical reconfigurations of their optical fields at the nanoscale. Other studies have shown ${ }^{15,34,35}$ the existence of long range interactions on disordered networks.

In this article, we experimentally study the optical field distribution on disordered plasmonic networks made of metal-dielectric films by far-field wavefront shaping. Non-local fluctuations of the field intensity mediated by plasmonic modes are observed. The spatial extent of these fluctuations is quantify with the introduction of an interaction length . This interaction length depends on the metal filling fraction and, in particular, show a clear 
increase around percolation that we relate to the existence of extended plasmonic modes. Correlation of these intensity fluctuations indicates that long-range interactions are mediated by a few modes only.

\section{Experimental methods}

In order to study these metal-dielectric films by means of wavefront shaping, an inverted microscope in leaky wave configuration ${ }^{36}$ is combined with a phase-only spatial light modulator (SLM) (Fig. 1(a)). A diode pumped solid state laser source at 785nm (DPSSL, Oxxius TM)

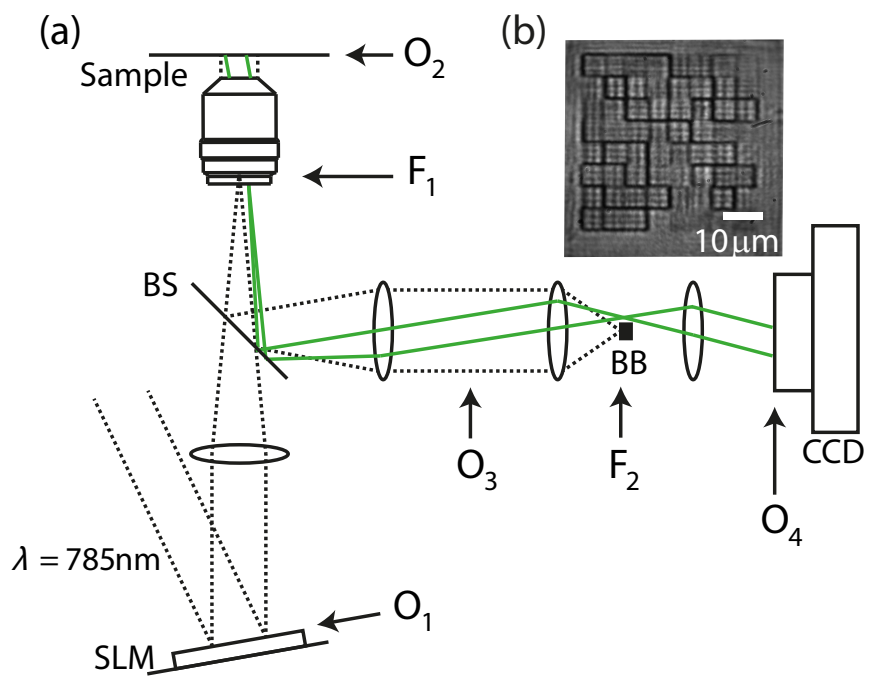

Figure 1: (a) Experimental set-up: light from a laser source (not shown) illuminates with a small incident angle $\left(<5^{\circ}\right)$ a phase-only SLM (Spatial Light Modulator). The latter is imaged on the sample surface with an oil-immersion objective $(\mathrm{NA}=1.4, \times 60)$. The same objective images the film surface on a CCD camera via a beam-splitter (BS). A beam block (BB) is installed in the Fourier plane of the sample surface along the detection path to remove ballistic light. $\mathrm{O}_{1}, \mathrm{O}_{2}, \mathrm{O}_{3}$ and $\mathrm{O}_{4}$ are the position of the object optical plane (i.e. sample plane), $F_{1}$ and $F_{2}$ are the position of the Fourier optical plane (back focal plane of the microscope objective) along the light path. (b) Image of a typical pattern of the SLM on the sample surface.

illuminates the SLM (Boulder Non Linear, 256x256 pixels) that allows up to $2 \pi$ phase-shift. Three lenses and an oil-immersion objective $(\mathrm{NA}=1.4, \times 60)$ image the SLM surface onto the sample plane with a $136 \times$ reduction. In this configuration, the illumination beam covers 
(a)

(b)
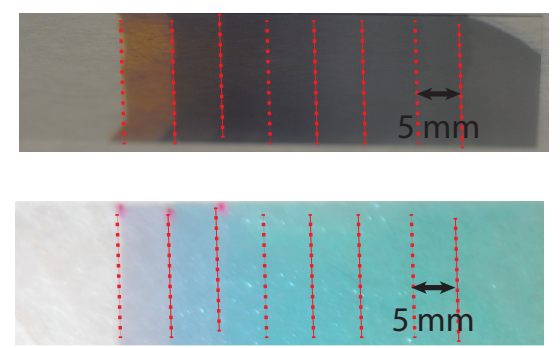

(c)

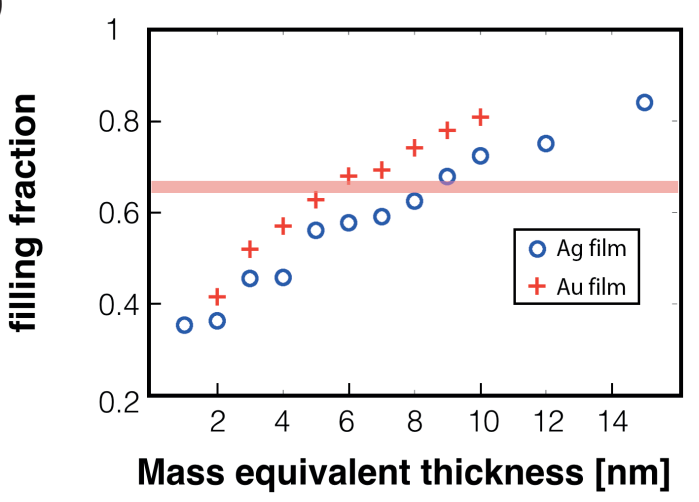

(d)

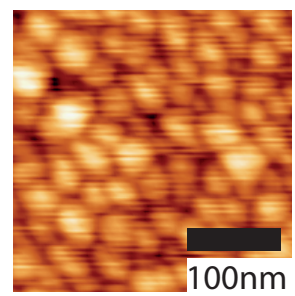

$36 \%$

(e)

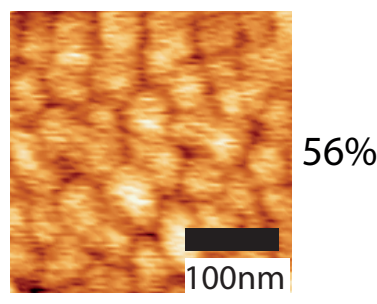

(f)

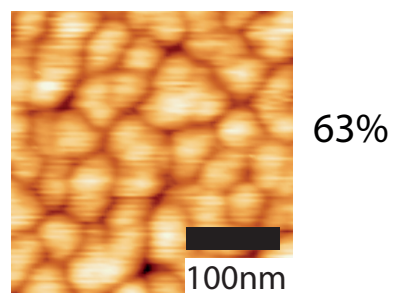

Figure 2: Photos of (a) silver and (b) gold films with increasing thickness from left (no metal) to right (almost continuous film). The vertical dashed lines delimits each incremental step in film thickness. The color change with film thickness is a characteristic of the specific light interaction with semicontinuous noble metal films around the percolation threshold. (c) Filling fraction with the mass equivalent thickness of metal, for gold and silver on glass substrate, measured from high resolution atomic force microscopy images. The horizontal bar shows the position where percolation threshold occurs. (d), (e) and (f) are $300 \times 300 \mathrm{~nm}^{2}$ high resolution atomic force microscopy images for silver films with filling fraction $\mathrm{ff}=0.36$ $(\mathrm{d}), \mathrm{ff}=0.56(\mathrm{e})$ and $\mathrm{ff}=0.63(\mathrm{f})$. (f) is around percolation. 
a $50 \mu \mathrm{m}$ radius area and one macro-pixel (the binning used in this experiment corresponds to $2 \times 2$ pixels of the SLM) covers $350 \times 350 \mathrm{~nm}^{2}$ on the sample surface, a surface slightly bigger than the one given by the diffraction limited spot size (280nm in the present configuration).

The sample is illuminated by the pattern imposed by the SLM and imaged on a CCD camera. A beam block in an intermediate Fourier plane along the detection path allows us to filter direct reflection from the sample. ${ }^{36}$ The samples under study are disordered metaldielectric films; they are naturally generated by electron-beam deposition of a thin layer of noble metals, such as gold or silver, on a glass substrate in a vacuum chamber. For a given deposition, the filling fraction is defined as $\mathrm{ff}=s_{m} /\left(s_{m}+s_{g}\right)$, where $s_{m}$ and $s_{g}$ are the surfaces covered by the metal and the glass respectively; it can be controlled by varying the amount of deposited material and characterised by AFM imaging. Figs. 2(a) and (b) show increasing thickness from pure glass to an almost continuous metal film, respectively for silver and gold. The change of color with the thickness is connected to a change in the filling fraction and the presence of a percolation threshold in the film that induces different plasmonic resonances. The increase of filling fraction with the mass equivalent thickness of the metal - i.e., the average thickness calculated from the deposited metal mass per surface unit - is shown in Fig. 2(c) for both gold and silver.

\section{Results and Discussion}

In disordered metal films, plasmonic modes can be easily coupled to the far field under any incidence. While previous works focused on the subwavelength features of these plasmonic modes, i.e. mainly their hot spots, there is no study that clearly quantifies the existence of bright extended modes with respect to the filling fraction on a scale larger than the wavelength. Yet, quantifying this information about these bright modes would be crucial for far-field control.

Disordered metal films are known to suffer from very strong scattering. The scattering that is not blocked by the beam block might prevent the detection of local variations, like 
those from plasmon scattering. Therefore, in order to get statistically relevant measurements of extended modes, we developed the following protocol: the film is illuminated with an SLM pattern uniform in amplitude but with a phase that can be spatially and temporally modulated. The phase pattern is chosen to be spatially random, with a local phase uniformly distributed between 0 and $2 \pi$. The spatial phase distribution over the lower part of the SLM pattern is kept constant, while the upper part of the SLM pattern is allowed to randomly evolve (Fig. 3(a)).

If the excitation of the optical fields on the films is only local, there should be no variation of the measured intensity in the area of the image corresponding to the lower part of the SLM pattern. Alternatively, any temporal fluctuation in this area should arise from non-local excitation coming from the upper part of the SLM pattern. In order to highlight fluctuations we calculated the variance of the intensity images $I(x, y, t)$, which is proportional to the variance of the modulated component of the electric field $E_{\text {mod }}(x, y, t)$ (see supplementary material):

$$
\sigma^{2}\left(E_{\text {mod }}(x, y, t)\right) \propto \frac{\sigma^{2}(I(x, y, t))}{I(x, y)}
$$

where $I(x, y)=\overline{I(x, y, t)}$ is the temporal average of the intensity. In fact, the local amplitude is the coherent sum of a stationary component due to the constant excitation from the lower part of the SLM pattern and a modulated component that we want to quantify coming from the upper part of the SLM pattern. In order to collect sufficient statistics for each filling fraction, we acquire a sequence of 100 images with a two-second integration time corresponding to different excitation phase patterns (Fig. 3(b)). Every image is normalised by the incident laser power.

The decay of the variance is computed as a function of the distance $x$ from the modulated part by averaging the variance image horizontally Fig. 3(c). This decay is represented by the doted line in (Fig. 3(c)), while the continuous line shows a fitting to an exponential function $e^{-x / L}$. In order to extract a relevant parameter to quantify the spatial extent of non-local excitations, we define the interaction length as the decay length $L$ of this exponential fitting. 
(a)

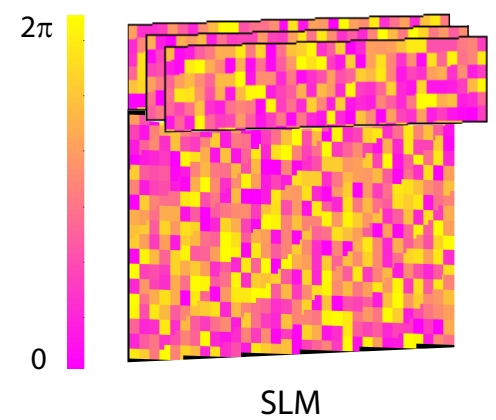

(b)

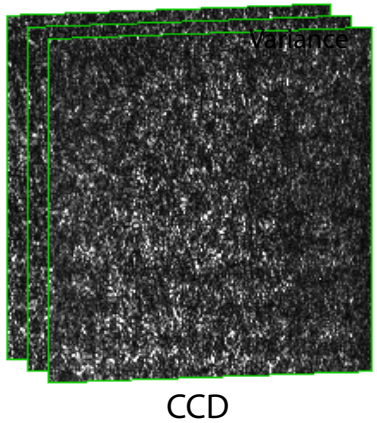

(c)

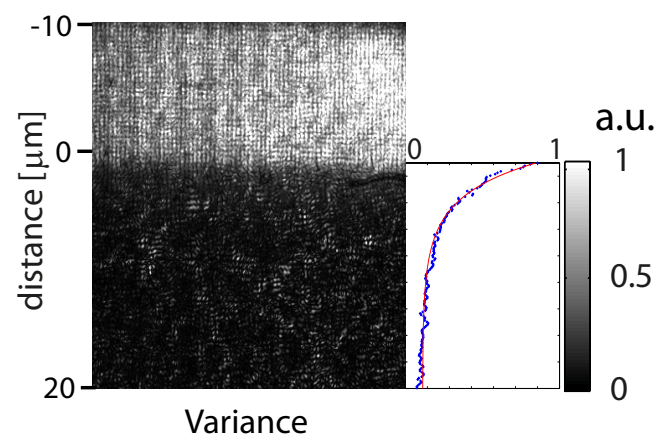

Figure 3: (a) Sequence of random SLM patterns, where only the top horizontal pattern varies. (b) CCD images of the sample under the random illumination patterns of the SLM shown in (a). (c) Normalised variance of 100 different CCD images. The upper band, i.e. the launching zone, corresponds to the part of the sample where the random incident illumination varies. On the far right: spatial average of the variance along the horizontal direction showing how the launching influences the lower part of the images. The red line is an exponential fit, from which the interaction length is extracted.

To evaluate the resolution limit of this measurement, we measured this decay length $L$ on continuous layer of copper thicker than $30 \mathrm{~nm}$, a non-plasmonic metal in the visible range, where no propagation is expected at all and obtained $L_{C u}=1.6 \mu \mathrm{m}$. We attribute this limit of resolution to scattering and spurious reflections of the optical setup. Above this limit, the values of $L$ correspond to long range interactions in the film. In the following, we study how $L$ varies as a function of the silver filling fraction ff (Figs.4(a)), well below percolation (ff $<0.5$ ), around percolation $(0.5<\mathrm{ff}<0.7)$ and above percolation (ff $>0.7) .{ }^{29}$ Error is evaluated by calculation of the variance at different areas of the sample. We also introduce a second parameter which plays an important role for non-local coupling, i.e. the number of modes contributing to the intensity fluctuations. At a given distance from the temporally modulated area, the image of the intensity correlation at the same point is calculated for different times. It gives information about the number of plasmonic modes that locally contribute to intensity variations. Close to the modulated region, we expect the correlation to be close to zero as many modes of different spatial extent contribute, while the correlation should reach values close to one very far away from the modulated zone because of lack 
of fluctuations. In particular, we decided to calculate the correlation $C_{L}$ at $L$ in order to quantify how many modes contribute to the interaction length $L$ (Figs.4(b)).
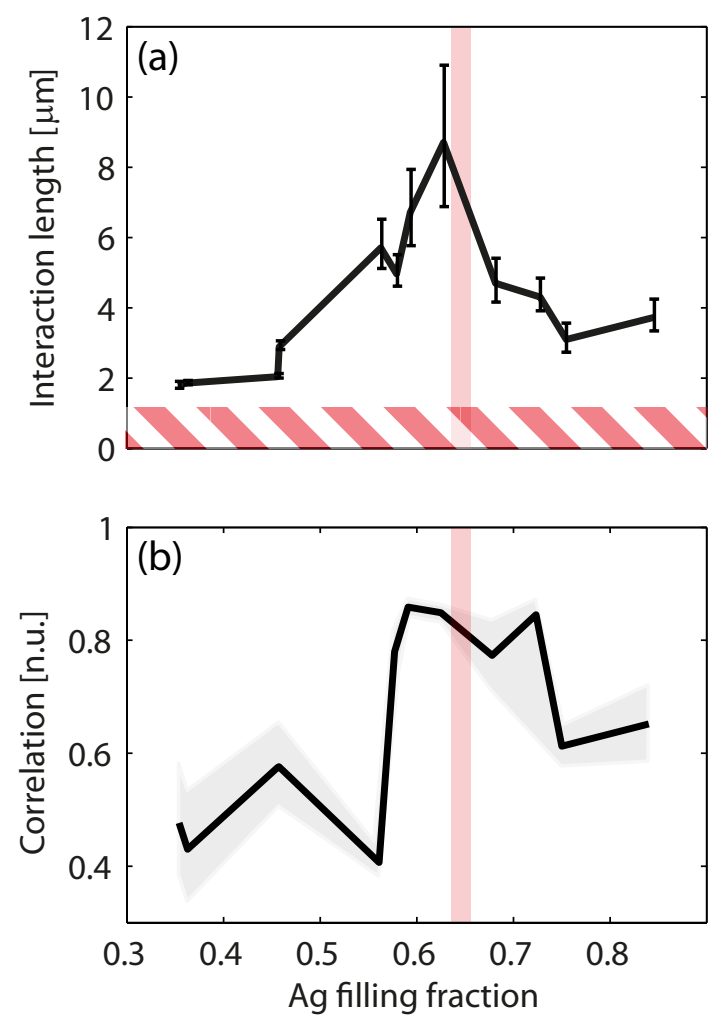

Figure 4: (a) Interaction length $L$ for different silver filling fractions ff. The horizontal dashed stripe shows the detection limit as measured on a continuous layer of copper. (b) Correlation of the intensity $C_{L}$ for different silver filling fractions ff. Error bars show measurement variance at different areas of the sample and the vertical line is the position of the percolation threshold.

Below percolation $(\mathrm{ff}<0.5)$ particles are far apart and modes are expected to be localised around metal aggregates.The measured interaction length is indeed around $2 \mu m$ in the order of the maximum particle size, and the correlation $C_{L}$ is around 0.5 indicating that several modes contribute at a shorter distance $L$. At high filling fractions (ff $>0.7$ ), when the metal layer is almost continuous and one could expect the presence of propagating surface plasmons (SPPs), the interaction length is longer and the correlation $C_{L}$ is of the order of 0.7 , showing that fewer long range modes are present: the values of $L$ are however lower than what is usually found with continuous silver films since, for the filling fractions used in this 
experiment, the metal film is still not continuous and its thickness is far from the optimum value for SPP propagation. ${ }^{37}$ The interesting regime is around percolation $(0.5 \leq \mathrm{ff} \leq 0.7),{ }^{29}$ where hot spots have been observed in the near field ${ }^{25}$ and extended modes theoretically predicted. ${ }^{38}$ Compared to the two other regimes, the interaction length $L$ shows a clear increase reaching an average of up to $8 \mu \mathrm{m}$ at $\mathrm{ff}=0.63$ where the correlation $C_{L}$ approaches 0.9. We interpret this result as the existence of only one dominant excited electromagnetic mode. Our correlation analysis is thus coherent with the decrease of the resonantly excited modes observed by Seal et al., ${ }^{26}$ although not equivalent to previous studies on disordered plasmonic film. ${ }^{27,29,39}$ The latter are dealing with the near-field regime where dark modes with very small extension ${ }^{32}$ are predominant, giving rise to the regime around percolation where modes are mostly localised. ${ }^{33}$ Here we probe bright modes whose extension has never been extensively studied experimentally on such random networks. $8 \mu \mathrm{m}$ is a surprisingly long interaction for silver at this wavelength. Yet Jess M. Gunn et al found much longer interactions with two photon luminescence on similar, although not identical, objects. ${ }^{34}$

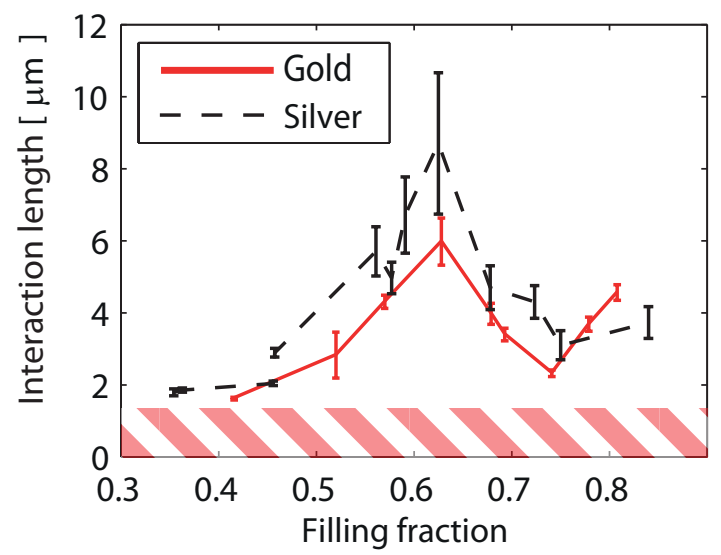

Figure 5: Comparison of the interaction length $L$ measured on gold and silver films for different filling fractions $\mathrm{ff}$. Percolation is around $\mathrm{ff}=0.65$. The shaded stripe shows the detection limit of our detection system as measured on a continuous layer of copper.

To verify the reproducibility and generality of this behaviour, we measured the interaction length on gold, a widely used plasmonic material, and compared these measurements with the previous on silver (Fig. 5). We observe a similar increase of the interaction length 
around percolation also for gold, although $L$ is lower in gold than in silver because of higher absorption at the experimental wavelength $\left(\operatorname{Re}\left[\epsilon_{\mathrm{Au}}\right]=-24\right.$ and $\left.\operatorname{Re}\left[\epsilon_{\mathrm{Ag}}\right]=-31^{40}\right)$.

\section{Conclusion}

We presented a method to characterise the non-local fluctuations of the field intensity on random plasmonic networks. This methodology can be readily adapted to other $2 \mathrm{D}$ and 3D random networks. In particular, the measured fluctuations on random plasmonic networks are associated with the existence of bright modes excited and detected from the far field. They correspond to a subpopulation of the total number of modes existing on these films. ${ }^{38}$ An important result of our analysis is the existence of long-range interactions around percolation that extend up to several micrometers. Correlation studies of these fluctuations point out a reduction of the number of bright modes around percolation. This is linked to the peculiar interaction of light with such films and could be analogous to the existence of a single extended mode on $2 \mathrm{D}$ random networks. ${ }^{24,41}$ The possibility of interacting with these extended modes from the far-field has important applications for quantum networks, coherent control and light-matter interactions at the nanoscale.

\section{Acknowledgement}

The authors acknowledge very helpful discussions with Rémi Carminati and Sébastien Bidault and the help from Emmanuelle Lacaze for the AFM images. This work was partially funded by the Agence Nationale de la Recherche under the grant ROCOCO project ANR-10-JCJC and by C'Nano Ile de France supported by Région Ile-de-France under the grant SNOOPY. This work was also supported by the European Research Council (Grant Number 278025). 


\section{Supporting Information Available}

In this section, we show that the time variance of the recorded intensity is related to the variance of the electric field at the sample surface. We used a beam block in the Fourier plane to filter direct reflection from the illumination on the surface. Therefore we record a fraction of the total scattered intensity. Since the sample is disordered on all scales, we can assume that the scattering is on average isotropic. We can therefore reasonable assume that the recorded intensity is simply proportional to the local intensity scattered from the surface. For any point $(x, y)$ of the sample plane, the electric field $E(x, y, t)$ is

$$
E(x, y, t)=E_{\mathrm{ref}}(x, y)+E_{\mathrm{mod}}(x, y, t)
$$

where $E_{\text {ref }}(x, y)$ is a stationary component and $E_{\text {mod }}(x, y, t)$ is a time varying component. In the lower part of the SLM pattern (Fig. 3), $E_{r e f}(x, y)$ is due to local excitation which is constant in time, while $E_{\text {mod }}(x, y, t)$ comes from non-local excitations from the time-varying part of the SLM pattern. With $I=|E|^{2}$, the intensity on the sample surface for one polarisation, for example along $x$, is :

$$
I_{x}(x, y, t)=I_{\mathrm{ref}, x}(x, y)+I_{\bmod , x}(x, y, t)+2 \operatorname{Re}\left[E_{\mathrm{ref}, x}(x, y) E_{\mathrm{mod}, x}^{*}(x, y, t)\right]
$$

If we assume $I_{\text {mod, } x}(x, y, t)<<I_{r e f, x}(x, y)$, a hypothesis that holds away from the upper part of the SLM pattern, the temporal variance of the intensity on the film is:

$$
\begin{aligned}
\sigma^{2}\left(I_{x}(x, y, t)\right)= & \sigma^{2}\left(I_{r e f, x}(x, y)\right)+\sigma^{2}\left(2 \operatorname{Re}\left[E_{r e f, x}(x, y) E_{\text {mod }, x}^{*}(x, y, t)\right]\right) \\
& +2 \sigma^{2}\left(I_{\text {ref }}(x, y), 2 \operatorname{Re}\left[E_{\text {ref }}(x, y) E_{\text {mod }}^{*}(x, y, t)\right]\right),
\end{aligned}
$$

where $\sigma^{2}(X, Y)=\overline{(X-\bar{X})(Y-\bar{Y})}$ is the covariance of $X$ and $Y, \sigma^{2}(X)=\sigma^{2}(X, X)$ is the variance of $X$ with $\bar{X}$ its mean value. As $E_{r e f, x}(x, y)$ is constant, $\sigma^{2}\left(I_{r e f, x}(x, y)\right)=0$ and, similarly, $\sigma^{2}\left(I_{\text {ref }, x}(x, y), 2 \operatorname{Re}\left[E_{\operatorname{ref}, x}(x, y) E_{\bmod , x}^{*}(x, y, t)\right]\right)=0$ because $E_{\bmod , x}^{*}(x, y, t)$ and 
$E_{\mathrm{ref}, x}(x, y)$ are independent. The variance of the intensity in Eq. 2 becomes

$$
\sigma^{2}\left(I_{x}(x, y, t)\right)=4 \sigma^{2}\left(\operatorname{Re}\left[E_{\mathrm{ref}, x}(x, y) E_{\mathrm{mod}, x}^{*}(x, y, t)\right]\right) .
$$

Writing explicitly $E_{\mathrm{ref}, x}(x, y)=E_{0, x} e^{i \phi_{0, x}}$ and $E_{\bmod , x}^{*}(x, y, t)=E_{1, x}(x, y, t) e^{-i \phi_{1, x}(x, y, t)}$, Eq. 3 simplifies as:

$$
\begin{aligned}
\frac{\sigma^{2}\left(I_{x}(x, y, t)\right)}{4} & =\sigma^{2}\left(\operatorname{Re}\left[E_{1, x}(x, y, t) e^{i\left(\phi_{0, x}-\phi_{1, x}(x, y, t)\right)}\right]\right) \times E_{0, x}^{2} \\
& =\sigma^{2}\left(\operatorname{Re}\left[E_{m o d, x}^{*}(x, y, t)\right]\right) \times E_{0, x}^{2}
\end{aligned}
$$

where $\phi_{0, x}$ is constant and, taking the temporal average of the intensity:

$$
\begin{aligned}
\overline{I_{x}(x, y, t)} & =\overline{I_{r e f, x}(x, y)}+2 \overline{\operatorname{Re}\left[E_{\text {ref }, x}(x, y) E_{\text {mod }, x}^{*}(x, y, t)\right]} \\
& =I_{r e f, x}(x, y)+2 \overline{\operatorname{Re}\left[E_{\text {ref }, x}(x, y) E_{\text {mod }, x}^{*}(x, y, t)\right]}
\end{aligned}
$$

When, as in our experiment, a large number of random excitation patterns is used, and since $\phi_{1, x}$ is normally distributed over $[0,2 \pi], \overline{\operatorname{Re}\left[e^{i\left(-\phi_{1, x}(x, y, t)\right)}\right]}=0$. Moreover, if we make the assumption that $E_{1, x}(x, y, t)$ varies little over time given the very low values in the lower part of Fig. 3(c), $\overline{\operatorname{Re}\left[E_{\text {ref }, x}(x, y) E_{\text {mod }, x}^{*}(x, y, t)\right]}=0$. Therefore, $\overline{I_{x}(x, y, t)}=I_{\text {ref }, x}(x, y)=E_{0, x}^{2}$. As a consequence,

$$
\frac{\sigma^{2}\left(I_{x}(x, y, t)\right)}{4}=\sigma^{2}\left(\operatorname{Re}\left[E_{\bmod , x}^{*}(x, y, t)\right]\right) \times \overline{I_{x}(x, y, t)} .
$$

Taking the amplitude of the electric field, we can write:

$$
\frac{\sigma^{2}\left(I_{x}(x, y, t)\right)}{4}=\sigma^{2}\left(E_{m o d, x}(x, y, t)\right) \times \overline{I_{x}(x, y, t)}
$$

Assuming that both in-plane polarisations are independent in metal-dielectric films ${ }^{41}$ we can generalise the previous equation to the total intensity as: 


$$
\sigma^{2}\left(E_{\text {mod }}(x, y, t)\right)=\frac{1}{2} \frac{\sigma^{2}(I(x, y, t))}{\overline{I(x, y, t)}}
$$

where the wavelength $\lambda$ is much larger than the average particle size $a$. Under this assumption the intensity variation in the direction of light propagation $z$ is confined along the film such as $I(x, y, z, t)=I(x, y, t) \cdot{ }^{24,27,42}$ Equation 8 clearly shows how the time variance of the intensity in the area corresponding to the lower part of the SLM in Fig. 3 is proportional to the time variance of the modulated electrical field. This main result of our derivation allows us to use intensity measurements as a mean to probe the time variance of the electric field at the sample surface. This material is available free of charge via the Internet at http://pubs.acs.org/.

\section{References}

(1) Brongersma, M. L.; Shalaev, V. M. The Case for Plasmonics. Science 2010, 328, 440441.

(2) Novotny, L.; van Hulst, N. Antennas for light. Nat Photon 2011, 5, 83-90.

(3) Anker, J. N.; Hall, W. P.; Lyandres, O.; Shah, N. C.; Zhao, J.; Van Duyne, R. P. Biosensing with plasmonic nanosensors. Nat Mater 2008, \%, 442-453.

(4) Atwater, H. A.; Polman, A. Plasmonics for improved photovoltaic devices. Nat Mater 2010, 9, 205-213.

(5) Tame, M. S.; McEnery, K. R.; Ozdemir, S. K.; Lee, J.; Maier, S. A.; Kim, M. S. Quantum plasmonics. Nat Phys 2013, 9, 329-340.

(6) Engheta, N. Circuits with Light at Nanoscales: Optical Nanocircuits Inspired by Metamaterials. Science 2007, 317, 1698-1702. 
(7) Hess, O.; Pendry, J. B.; Maier, S. A.; Oulton, R. F.; Hamm, J. M.; Tsakmakidis, K. L. Active nanoplasmonic metamaterials. Nat Mater 2012, 11, 573-584.

(8) Fang, N.; Lee, H.; Sun, C.; Zhang, X. Sub-Diffraction-Limited Optical Imaging with a Silver Superlens. Science 2005, 308, 534-537.

(9) Stockman, M. I.; Faleev, S. V.; Bergman, D. J. Coherent Control of Femtosecond Energy Localization in Nanosystems. Phys. Rev. Lett. 2002, 88, 067402.

(10) Aeschlimann, M.; Bauer, M.; Bayer, D.; Brixner, T.; García de Abajo, F. J.; Pfeiffer, W.; Rohmer, M.; Spindler, C.; Steeb, F. Adaptive subwavelength control of nano-optical fields. Nature 2007, 446, 301-4.

(11) Volpe, G.; Cherukulappurath, S.; Juanola Parramon, R.; Molina-Terriza, G.; Quidant, R. Controlling the optical near field of nanoantennas with spatial phaseshaped beams. Nano letters 2009, 9, 3608-11.

(12) Volpe, G.; Molina-Terriza, G.; Quidant, R. Deterministic Subwavelength Control of Light Confinement in Nanostructures. Physical Review Letters 2010, 105, 216802.

(13) Gjonaj, B.; Aulbach, J.; Johnson, P. M.; Mosk, A. P.; KuipersL.,; Lagendijk, A. Active spatial control of plasmonic fields. Nat Photon 2011, 5, 360-363.

(14) Kao, T. S.; Rogers, E. T. F.; Ou, J. Y.; Zheludev, N. I. "Digitally" Addressable Focusing of Light into a Subwavelength Hot Spot. Nano Letters 2012, 12, 2728-2731.

(15) Aeschlimann, M.; Brixner, T.; Cunovic, S.; Fischer, A.; Melchior, P.; Pfeiffer, W.; Rohmer, M.; Schneider, C.; Strüber, C.; Tuchscherer, P.; Voronine, D. Nano-Optical Control of Hot-Spot Field Superenhancement on a Corrugated Silver Surface. IEEE Journal of Selected Topics in Quantum Electronics 2012, 18, 275-282.

(16) Wenseleers, W.; Stellacci, F.; Meyer-Friedrichsen, T.; Mangel, T.; Bauer, C. A.; Pond, S. J. K.; Marder, S. R.; Perry, J. W. Five Orders-of-Magnitude Enhancement of Two- 
Photon Absorption for Dyes on Silver Nanoparticle Fractal Clusters. The Journal of Physical Chemistry B 2002, 106, 6853-6863.

(17) Sanchot, A.; Baffou, G.; Marty, R.; Arbouet, A.; Quidant, R.; Girard, C.; Dujardin, E. Plasmonic Nanoparticle Networks for Light and Heat Concentration. ACS Nano 2012, $6,3434-3440$.

(18) Gaio, M.; Castro-Lopez, M.; Renger, J.; van Hulst, N.; Sapienza, R. Percolating plasmonic networks for light emission control. Faraday Discuss. 2015, 178, 237-252.

(19) Granqvist, C.; Hunderi, O. Optical properties of ultrafine gold particles. Physical Review $B 1977,16$.

(20) Yagil, Y.; Yosefin, M.; Bergman, D.; Deutscher, G.; Gadenne, P. Scaling theory for the optical properties of semicontinuous metal films. Physical Review B 1991, 43, 342-352.

(21) Sarychev, A. K.; Shalaev, V. M. Electromagnetic field fluctuations and optical nonlinearities in metal-dielectric composites. Physics Report 2000, 335, 275-371.

(22) Stockman, M.; Pandey, L.; Muratov, L.; George, T. Giant fluctuations of local optical fields in fractal clusters. Physical Review Letters 1994, 72, 1-4.

(23) Brouers, F.; Blacher, S.; Sarychev, A. Giant field fluctuations and anomalous light scattering from semicontinuous metal films. Physical Review B 1998, 58, 15897-15903.

(24) Shalaev, V., Ed. Optical properties of nanostructured random media; Topics in Applied Physics; Springer Verlag, 2002; Vol. 82; pp 169-213.

(25) Grésillon, S.; Aigouy, L.; Boccara, A. C.; Rivoal, J.; Quelin, X.; Desmarest, C.; Gadenne, P.; Shubin, V.; Sarychev, A.; Shalaev, V. Experimental Observation of Localized Optical Excitations in Random Metal-Dielectric Films. Physical Review Letters 1999, 82, 4520-4523. 
(26) Seal, K.; Sarychev, A.; Noh, H.; Genov, D.; Yamilov, A.; Shalaev, V.; Ying, Z.; Cao, H. Near-Field Intensity Correlations in Semicontinuous Metal-Dielectric Films. Physical Review Letters 2005, 94, 226101.

(27) Krachmalnicoff, V.; Castanié, E.; De Wilde, Y.; Carminati, R. Fluctuations of the Local Density of States Probe Localized Surface Plasmons on Disordered Metal Films. Physical Review Letters 2010, 105, 183901.

(28) Word, R. C.; Dornan, T.; Könenkamp, R. Photoemission from localized surface plasmons in fractal metal nanostructures. Applied Physics Letters 2010, 96, 251110.

(29) Awada, C.; Barbillon, G.; Charra, F.; Douillard, L.; Greffet, J.-J. Experimental study of hot spots in gold/glass nanocomposite films by photoemission electron microscopy. Physical Review B 2012, 85, 045438.

(30) Pavaskar, P.; Hsu, I.-K.; Theiss, J.; Hung, W. H.; Cronin, S. B. A microscopic study of strongly plasmonic Au and Ag island thin films. Journal of Applied Physics 2013, 113, 034302.

(31) Borys, N. J.; Shafran, E.; Lupton, J. M. Surface plasmon delocalization in silver nanoparticle aggregates revealed by subdiffraction supercontinuum hot spots. Scientific Reports 2013, 3, 2090.

(32) Cazé, A.; Pierrat, R.; Carminati, R. Radiative and non-radiative local density of states on disordered plasmonic films. Photonics and Nanostructures - Fundamentals and Applications 2012, 10, 339-344.

(33) Cazé, A.; Pierrat, R.; Carminati, R. Spatial Coherence in Complex Photonic and Plasmonic Systems. Physical Review Letters 2013, 110, 063903.

(34) Gunn, J. M.; Ewald, M.; Dantus, M. Polarization and Phase Control of Remote 
Surface-Plasmon-Mediated Two-Photon-Induced Emission and Waveguiding. Nano Letters 2006, 6, 2804-2809.

(35) Gunn, J. M.; High, S. H.; Lozovoy, V. V.; Dantus, M. Measurement and Control of Ultrashort Optical Pulse Propagation in Metal Nanoparticle-Covered Dielectric Surfaces. The Journal of Physical Chemistry C 2010, 114, 12375-12381.

(36) Bouhelier, A.; Wiederrecht, G. P. Surface plasmon rainbow jets. Optics letters 2005, $30,884-6$.

(37) Berini, P. Plasmon-polariton waves guided by thin lossy metal films of finite width: Bound modes of asymmetric structures. Phys. Rev. B 2001, 63, 125417.

(38) Stockman, M.; Faleev, S.; Bergman, D. Localization versus Delocalization of Surface Plasmons in Nanosystems: Can One State Have Both Characteristics? Physical Review Letters 2001, 87, 167401.

(39) Seal, K.; Genov, D.; Sarychev, A.; Noh, H.; Shalaev, V.; Ying, Z.; Zhang, X.; Cao, H. Coexistence of Localized and Delocalized Surface Plasmon Modes in Percolating Metal Films. Physical Review Letters 2006, 97, 206103.

(40) Johnson, P.; Christy, R. Optical constants of the noble metals. Physical Review B 1972, 1318.

(41) Ducourtieux, S.; Podolskiy, V. A.; Grésillon, S.; Buil, S.; Berini, B.; Gadenne, P.; Boccara, A. C.; Rivoal, J. C.; Bragg, W. D.; Banerjee, K.; Safonov, V. P.; Drachev, V. P.; Ying, Z. C.; Sarychev, A. K.; Shalaev, V. M. Near-field optical studies of semicontinuous metal films. Phys. Rev. B 2001, 64, 165403.

(42) Brouers, F.; Blacher, S.; Lagarkov, A. N.; Sarychev, A. K.; Gadenne, P.; Shalaev, V. M. Theory of giant Raman scattering from semicontinuous metal films. Phys. Rev. B 1997, $55,13234-13245$. 